\title{
PAPER
}

\section{Long term treatment and disease severity change brain responses to levodopa in Parkinson's disease}

\author{
T Hershey, K J Black, J L Carl, L McGee-Minnich, A Z Snyder, J S Perlmutter
}

J Neurol Neurosurg Psychiatry 2003;74:844-851

See end of article for authors' affiliations

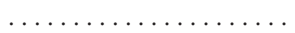

Correspondence to: Dr J S Perlmutter, Campus Box 8225,4525 Scott Avenue, St Louis, MO 63110, USA joel@npg.wustl.edu

Received 25 July 2002 Accepted in final revised form

4 February 2003 Objectives: Degeneration of nigrostriatal neurons and subsequent striatal dopamine deficiency
produce many of the symptoms of Parkinson disease (PD). Initially restoration of striatal dopamine with produce many of the symptoms of Parkinson disease (PD). Initially restoration of striatal dopamine with
oral levodopa provides substantial benefit, but with long term treatment and disease progression, levodopa can elicit additional clinical symptoms, reflecting altered effects of levodopa in the brain. The authors examined whether long term treatment affects the brain's response to levodopa in the absence of these altered clinical responses to levodopa.

Methods: Positron emission tomography (PET) measurements were used of brain-blood flow before and after an acute dose of levodopa in three groups: PD patients treated long term with levodopa without levodopa induced dyskinesias, levodopa naive PD patients, and controls.

Results: It was found that the PD group treated long term responded to acute levodopa differently from controls in left sensorimotor and left ventrolateral prefrontal cortex. In both regions, the treated PD group had decreased blood flow whereas the control group had increased blood flow in response to levodopa. Levodopa naive PD patients had little or no response to levodopa in these regions. Within the treated PD group, severity of parkinsonism correlated with the degree of abnormality of the sensorimotor cortex response, but not with the prefrontal response.

Conclusions: It is concluded that long term levodopa treatment and disease severity affect the physiology of dopaminergic pathways, producing altered responses to levodopa in brain regions associated with motor function.

D egeneration of nigrostriatal neurons with subsequent deficiency of striatal dopamine produces many of the clinical manifestations of Parkinson disease (PD). Initially, oral levodopa, the immediate precursor of dopamine, improves many of the symptoms associated with the disease. Once transported across the blood-brain barrier, levodopa is converted to dopamine and preferentially accumulates in the striatum. ${ }^{1}$ However, as the disease progresses and long term treatment continues, the behavioural response to levodopa changes. For example, after years of levodopa treament patients may develop involuntary movements (levodopa induced dyskinesias), mood fluctuations, increased cognitive impairment, or psychosis in response to levodopa. ${ }^{23}$ Although some studies of chronically treated rodents with nigrostriatal lesions support the notion that long term treatment causes these changes in response to levodopa, ${ }^{4}$ other observations indicate that severity of disease is an additional factor. ${ }^{5-7}$

Exposure to levodopa or other dopamine agonists may begin to change the neurophysiology of the dopamine pathways before any obvious accompanying change in behaviour. ${ }^{48}$ These changes may also be influenced by the severity of the disease. The purpose of this investigation is to determine whether long term exposure to levodopa in humans is associated with altered regional brain responses to a dopaminergic challenge in the absence of changes in the behavioural response to levodopa.

We use positron emission tomography (PET) to measure blood flow responses to an oral levodopa challenge as an indicator of the function of dopamine mediated pathways. Local blood flow or metabolic responses to behavioural or pharmacological challenges primarily reflect changes in axonal terminal fields or local interneurons ${ }^{9-11}$ rather than local vascular changes. ${ }^{12-14}$ Specifically, we have shown previously that with adequate carbidopa pretreatment, levodopa does not produce a significant vascular response. ${ }^{14} 15$ Blood flow responses to dopaminergic challenges can determine how regions of the brain downstream from dopamine receptors are affected in diseases such as $\mathrm{PD}^{16-22}$ and how any alterations in function relate to clinical symptoms. ${ }^{1523}$ Our hypothesis was that long term treatment with levodopa would change the neuronal response to an acute levodopa challenge in PD.

\section{METHODS \\ Subjects}

Patients with clinically diagnosed idiopathic PD $(n=28)$ were recruited from the Movement Disorders Center at Washington University School of Medicine. Patients were excluded from the study for any evidence of secondary parkinsonism (for example, drug induced or atypical presentation), dementia (Mini-Mental Status Exam (MMSE) score <26), ${ }^{24}$ depression (Hamilton Scale score >10), ${ }^{25}$ history of levodopa induced dyskinesias or psychosis, history of other neurological disorders, psychiatric disorders, substance misuse, neuroleptic use, or suspicion of pregnancy. Patients either were chronically treated with levodopa (treated PD; $n=12$; average daily dose of levodopa $=452 \mathrm{mg}, \mathrm{SD}=31 \mathrm{mg}$ ) or had no history of levodopa or other dopamine agonist treatment (levodopa naive $\mathrm{PD} ; \mathrm{n}=16$ ). Three of the 12 treated PD patients were also taking an additional dopamine agonist (one subject taking each of the following: pramipexole, bromocriptine, and pergolide). Normal controls also were scanned (controls; $\mathrm{n}=16)$. The three groups did not differ significantly in age (table 2, $F(2,41)=2.41, \mathrm{p}=0.10$ ), however, the control group was on average younger than the two PD groups. All subjects were right handed. Eight of the 12 treated PD patients and 8 of the 16 levodopa naive PD patients had greater symptoms on

Abbreviations: PD, Parkinson's disease; PET, positron emission tomography 
Table 1 Demographic and clinical variables

\begin{tabular}{|c|c|c|c|c|}
\hline & \multirow[b]{2}{*}{$\begin{array}{l}\text { Normals } \\
(n=16)\end{array}$} & \multicolumn{2}{|l|}{ PD groups } & \multirow[b]{2}{*}{$\mathrm{p}$ value } \\
\hline & & $\begin{array}{l}\text { Levodopa naive } \\
(n=16)\end{array}$ & Treated $(n=12)$ & \\
\hline Age & 57.9 (13.7) & $60.9(12.3)$ & $67.7(6.4)$ & 0.10 \\
\hline Hamilton Depression Scale & $1.0(1.5)$ & $2.4(2.4)$ & $1.3(2.6)$ & 0.22 \\
\hline Mini-Mental Status Score & $29.5(0.9)$ & $29.2(0.7)$ & $28.8(1.2)$ & 0.22 \\
\hline Baseline Modified UPDRS* & & $4.4(3.2)$ & $8.1(5.4)$ & 0.03 \\
\hline Symptom duration $(y)$ & & $3.0(2.9)$ & $7.2(6.0)$ & 0.02 \\
\hline Treatment duration $(y)$ & & & $5.5(5.4)$ & \\
\hline Modified UPDRS $\%$ change after acute dose of levodopa & & $-39.9(64.2)$ & $-28.7(50.1)$ & 0.62 \\
\hline
\end{tabular}

the right side of the body. Four of the 12 treated PD patients noted slight diminution of motor benefit before a dose of levodopa, but none were taking levodopa more than four times per day. The two PD groups differed significantly in symptom duration and baseline modified motor Unified Parkinson Disease Rating Scale (UPDRS), ${ }^{26}$ but not in Mini-Mental Status examination scores or change in UPDRS with levodopa (table 1). As a whole, PD patients demonstrated significant improvement in motor symptoms with levodopa at the time of the post-levodopa PET scans (modified UPDRS scale, mean percentage change $=35 \%$ ).

All subjects provided written informed consent before participation in the study. The study protocol was approved by the Radioactive Drug Research Committee and the Human Studies Committee of Washington University School of Medicine. A subset of these scans have been previously compared with scans from PD patients with levodopa induced dyskinesias. ${ }^{15}$

\section{Protocol}

We performed PET scans on PD patients and controls at baseline and after an oral dose of levodopa. The specific methods are as follows:

\section{PET}

PET studies were performed in 2D mode on a Siemens 953B or 961 HR scanner (CTI, Knoxville, TN). Only 9 of the 44 subjects were scanned on the 961 scanner (five controls, two treated $\mathrm{PD}$, and two levodopa naive PD subjects). On the 953B scanner, data were recorded simultaneously for 31 slices with a centre to centre slice separation of $3.4 \mathrm{~mm}$. Axial and transaxial spatial resolution was about $4.5 \mathrm{~mm}$ full width at half maximum (FWHM) at slice centre in $2 \mathrm{D}$ mode. ${ }^{27}$ On the 961 scanner, data were recorded simultaneously for 47 slices with a $3.25 \mathrm{~mm}$ centre to centre slice separation. In plane transaxial and axial spatial resolutions were about $4 \mathrm{~mm}$ FWHM at slice centre in 2D wobbled mode. There are no gaps in data collection with either scanner. After subjects were positioned, a transmission scan used for individual attenuation correction was acquired with rotating rod sources containing ${ }^{68} \mathrm{Ge} /{ }^{68} \mathrm{Ga}^{27}$ Images from both scanners were smoothed to the same in-plane pixel size of $2.086 \mathrm{~mm}^{2}$ We then apply 3D smoothing ( $14 \mathrm{~mm}$ FWHM) to images from both scanners to obtain identical axial and transaxial resolutions. We then resample these 3D image sets after transformation into stereotactic coordinates in Talairach space. Finally, we normalise mean counts on a scan by scan basis to control for minor differences in the global counts achieved. These last steps make any small intrinsic differences in sampling, sensitivity, and resolution negligible. Blood flow was measured using a 40 second emission scan after the intravenous bolus injection of $5-10 \mathrm{ml}$ of saline containing $40-50 \mathrm{mCi}$ of ${ }^{15} \mathrm{O}$ labelled water. ${ }^{28}$

PD subjects chronically treated with levodopa refrained from taking levodopa for at least 12 hours before the PET scans ("practical off"). On the morning before their scan, all subjects had a baseline clinical evaluation including UPDRS ratings, and then took $200 \mathrm{mg}$ carbidopa orally. Subjects were placed in the scanner with an individually molded polyform mask to help minimise head movement. A 20 gauge catheter was inserted into an antecubital vein to permit injection of $\mathrm{H}_{2}{ }^{15} \mathrm{O}$. Some subjects also had a similar catheter inserted into the radial artery at the wrist after local lidocaine anaesthesia for arterial blood sampling.

Once these preparatory steps were completed, we performed two to three baseline 40 second PET measurements of blood flow 15 minutes apart. ${ }^{29}$ We then gave subjects levodopa/carbidopa (150 mg/37.5 mg) orally. About 45-75 minutes after levodopa/carbidopa administration, we collected two to three more 40 second PET measurements of blood flow 15 minutes apart. At the beginning of this study, we performed two baseline and two post-levodopa scans, and waited 75 minutes after levodopa before beginning to scan again. However, after obtaining levodopa plasma concentrations from the first set of subjects, we realised that plasma concentrations peaked between 30 and 45 minutes after levodopa administration and remained comparatively high for longer than anticipated. Thus, we revised our protocol to acquire three baseline and three post-levodopa PET scans, and only waited 45 minutes after adminstration before beginning to scan again. Importantly, levodopa levels at the time of the scans did not differ across groups and although subjects varied in the number of total scans performed (between four and six), we analysed a single contrast image (baseline compared with on levodopa scans) for each subject. In this manner, each subject contributed equally to our statistical analyses.

During each PET scan, the room was darkened and quiet, subjects' eyes were closed, and subjects remained still. In between PET scans, we performed clinical ratings and obtained blood samples. The clinical ratings occurred after every scan, and consisted of a modified version of the motor subscale 3 from the UPDRS (ratings for tremor, rigidity, bradykinesia and tapping speed for upper extremities; 16 total possible points for each side, 32 points total). Blood samples were taken once before levodopa/carbidopa administration and then every 15 minutes after levodopa/carbidopa administration including samples done immediately after each subsequent PET scan. No clinical ratings or blood samples were performed during blood flow measurements. Each subject also had a high resolution anatomical MRI scan performed on the same day as the PET.

\section{Levodopa measurements}

Levodopa and carbidopa levels were measured using high performance liquid chromatography with electrochemical detection following a modified version of published methods. ${ }^{30}$ We added an internal standard, 3,4-dihydrobenzylamine (DHBA), to simplify quantification. ${ }^{31}$

\section{PET analysis}

Data from both scanners were combined. Although the field of view was larger from the 961 PET scanner, we only analysed 

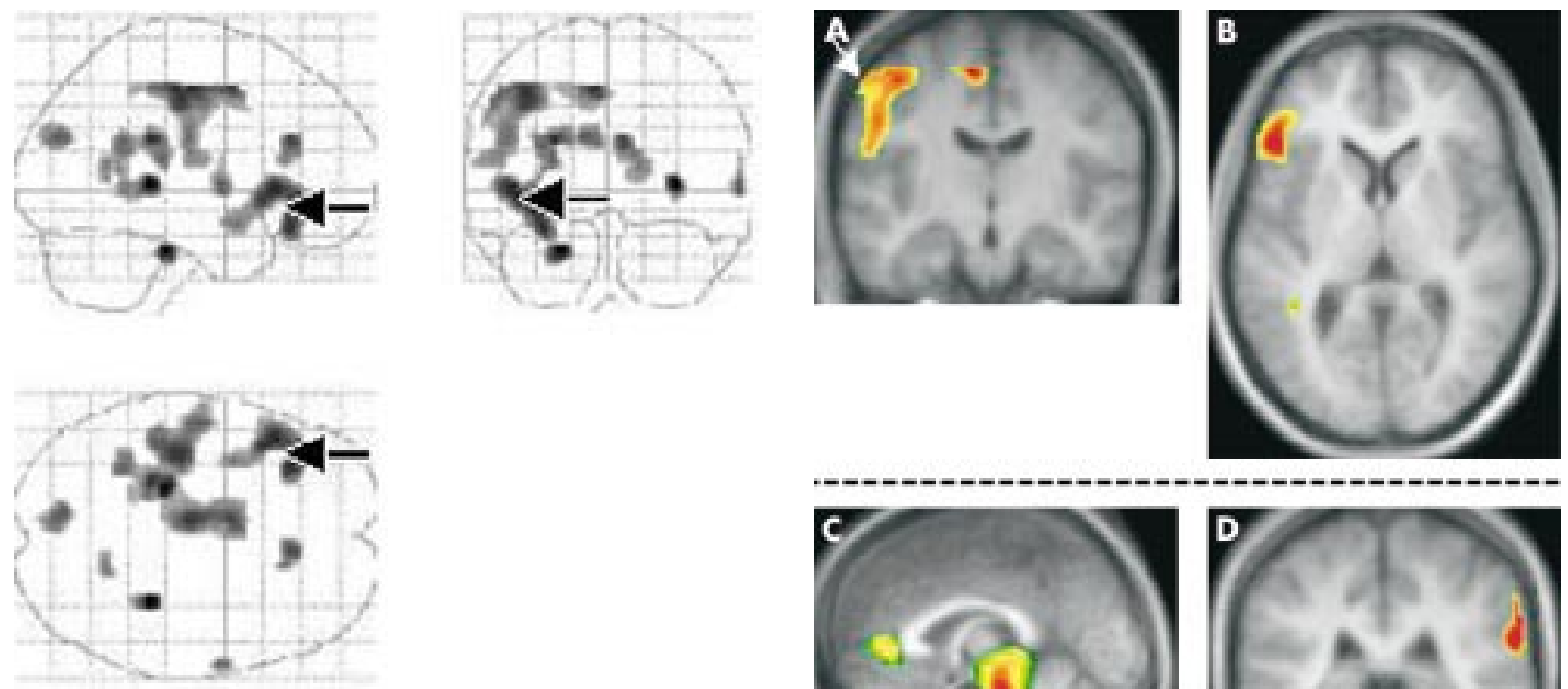

Figure 1 "Glass brain" views of the comparison of levodopa induced responses in normal subjects compared with treated PD patients for $t=2.5$ threshold. No cluster threshold has been set to better illustrate the field of view of the data. The black arrow points to a coordinate within the left VLPFC.

data from slices to which all subjects contributed. The brain coverage is best displayed in the sagittal view of figure 1. PET images first were aligned to individual subject MRIs and transformed to Talairach stereotactic space using previously described methods. ${ }^{32-37}$ This process was performed before images were imported into SPM. A person's PET images were aligned to each other, then the averaged PET image for that person was warped to that person's MRI scan using an 8 parameter transformation. Next, the MRI was warped to an averaged MRI already in Talairach atlas space (consisting of 12 normal adult brains) using a 12 parameter affine transformation. Using this transformation matrix, we then transformed the person's PET directly into atlas space. ${ }^{34}$ Scans were normalised by setting mean qualitative blood flow for each scan to 1000. Spatial smoothing was done with a $14 \mathrm{~mm}$ gaussian filter. Effective resolution as determined by SPM was $\mathrm{x}=14 \mathrm{~mm}, \mathrm{y}=16 \mathrm{~mm}$, and $\mathrm{z}=17 \mathrm{~mm}$.

To statistically analyse the PET images, we used a random effects analysis implemented by the freely available SPM99 software package ${ }^{38}$ (http://www.fil.ion.ucl.ac.uk/spm/spm99. html). With this analysis, we examined the entire brain volume to determine where blood flow responses differed between groups, and where they were consistent across all subjects while providing adequate protection against type I error. Because of the rigour of this procedure, there may be increased type II error and modest effects may not be detected. However, this procedure strengthens the conclusions that can be drawn from group comparisons and has been recommended for groups of more than 12-16 members. ${ }^{39}$

In random effects analyses, first a contrast image is made for each person to represent change in blood flow at each voxel from the baseline to the levodopa condition. Next, these contrast images were analysed with two sample $t$ tests to identify differences in the response to levodopa between two groups (between group comparison; treated PD compared with controls, levodopa naive PD compared with controls, treated PD compared with levodopa naive PD) or a one sample $t$ test to find significant areas of response to levodopa across all subjects. A $t$ value was assigned to each voxel in the brain. These $t$ maps were examined for voxels that exceeded a height threshold ( $t=2.5$ for between group comparison or $t=3.0$ for the single group comparison). Clusters of these voxels that exceeded an extent threshold (set at 30) were identified. A
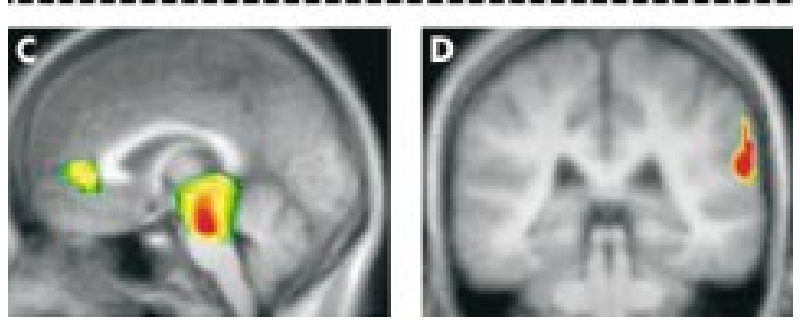

Figure 2 SPM99 display of voxel clusters that demonstrated significant effects (in colour) overlaid on a composite MRI (black and white) in atlas space. Voxels shown survived the height and cluster size threshold. However, not all clusters in the images survived the cluster level multiple comparison correction. (A) Coronal view of the left sensorimotor cortex region (SMC; indicated by arrow). Treated PD and controls responded significantly differently to levodopa in this region. The midline region that also appears in this image was not significant after correction for multiple comparisons. (B) Horizontal view of the left ventrolateral prefrontal cortex (VLPFC) region. Treated PD and controls responded significantly differently to levodopa in this region. (C) Sagittal view of the anterior cingulate and midbrain regions. A significant increase in blood flow after an acute dose of levodopa occurred in these regions, averaged across all subjects. (D) Coronal view of the right lateral inferior parietal region. A significant decrease in blood flow after an acute dose of levodopa occurred in this region, averaged across all subjects.

multiple comparison correction for number of possible clusters of this size and magnitude in the brain volume was applied. Clusters that reached a corrected $p$ value of less than 0.05 then were considered for further analysis and interpretation.

The regions defined by these statistically determined clusters then were applied to the smoothed, normalised individual subjects' images to obtain mean blood flow values within the clusters from the individual scans. Mean voxel values for the before and on levodopa conditions were calculated for each subject for each region. Comparisons were performed on these mean blood flow values using independent samples $t$ tests to determine the extent and direction of effects across conditions and groups. Pearson correlation coefficients were calculated between relevant clinical variables (UPDRS score, symptom duration, and treatment duration) and blood flow responses within groups for the significant clusters. Global blood flow values were analysed with a repeated measures general linear model, with group as the independent variable and condition (baseline, on levodopa) as the repeated measure.

\section{RESULTS}

\section{Between groups analysis}

SPM99 whole brain analyses revealed significant differences in the regional responses to a levodopa challenge between control and treated PD groups. SPM99 whole brain analyses did not reveal any significant differences between control and levodopa naive PD groups or between treated and levodopa 

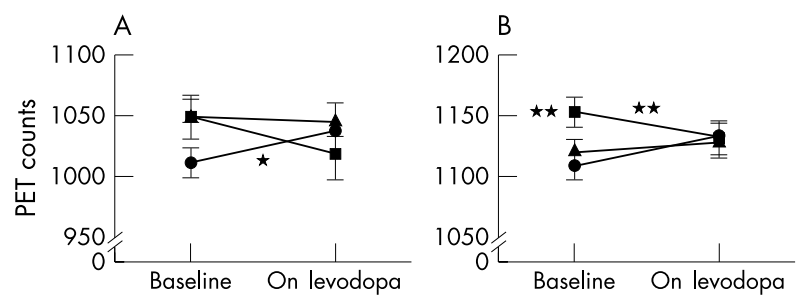

Figure 3 Means (SEM) for baseline and on levodopa blood flow in (A) left SMC and (B) left VLPFC for control group (circles), levodopa naive PD group (triangles); and treated PD group (squares). ${ }^{*}$ In the left SMC, change in blood flow with levodopa in the control group was significantly different from the two PD groups $(p<0.05) .{ }^{* *} \ln$ the left VLPFC, baseline blood flow and change in blood flow with levodopa in the treated PD group were significantly different from the other two groups $(p<0.05)$.

naive PD groups. The regions that were significantly different between control and treated PD groups were located in the left sensorimotor cortex (SMC) and left ventrolateral prefrontal cortex (VLPFC) (see table 2). The SMC region included both sides of the central sulcus along the superior-inferior extent (fig 2A). However, the most superior portion of the SMC was not sampled because of our limited field of view. The VLPFC region extended from Brodmann regions 44, 45, and 46 superiorly to areas 47 and the posterior portion of area 10 inferiorly (fig 2B). To characterise differences between groups and to determine the clinical relevance of these responses, we performed further analyses on the blood flow responses in these two regions.

\section{SMC region}

Figure 3A shows the differences in the SMC blood flow response to levodopa across the three groups. At baseline, the two PD groups were not significantly different from each other, but both were different from controls at a nonsignificant level (levodopa naive PD $v$ controls, $t=-1.8$, $\mathrm{p}=0.08$, treated PD $v$ controls, $t=-1.8, \mathrm{p}=0.08)$. On levodopa, the groups did not differ significantly in mean blood flow for the SMC region. However, the change in blood flow in response to levodopa was different between the treated PD group and the controls $(t=3.5, \mathrm{p}=0.002)$. The treated PD group had decreased blood flow in this region whereas the control group had increased blood flow. Interestingly, the degree of change between baseline and levodopa conditions for the levodopa naive PD group (nearly zero) was intermediate between the treated PD group and the controls, but was significantly different from the controls only (dopa naive PD $v$ treated PD, $t=1.7, \mathrm{p}=0.11$; levodopa naive PD $v$ controls, $t=2.8$, $\mathrm{p}=0.009$ ). Within group analyses (one sample $t$ tests) revealed that the control group had a significant increase in blood flow $(t=3.2, \mathrm{p}=0.006)$ and the treated PD group had a nonsignificant decrease in blood flow in the left SMC region $(t=-1.9, \mathrm{p}=0.08)$. The levodopa naive PD group did not have a significant response in the SMC $(t=-0.3, p=0.72)$ (see fig $3 \mathrm{~A}$ and $4 \mathrm{~A})$.
Age did not correlate significantly with response in this region, either within or across groups. Furthermore, we removed four of the youngest normal controls to provide a better age matched subsample for the treated PD group. This modification eliminated the age difference between the two groups (normal control mean age $=64.3, \mathrm{SD}=6.8$; treated $\mathrm{PD}$ mean age $=67.7, \mathrm{SD}=6.4 ; t=-1.2, \mathrm{p}=0.23$ ), but did not change the difference in blood flow response to levodopa in the SMC $(t=3.2, \mathrm{p}=0.004)$.

Within the treated PD group, left SMC response correlated with baseline modified UPDRS ( $r=-0.72, \mathrm{p}=0.008)$, such that patients with greater motor severity showed greater reduction of blood flow to levodopa in this region. This correlation was not significant in the levodopa naive patients $(r=-0.06$, $\mathrm{p}=0.83$ ), but these patients were also less severely affected on average than the treated PD group. Covarying treatment duration (partial $r=-0.76, \mathrm{p}=0.007$ ) or symptom duration (partial $r=-0.73, \mathrm{p}=0.001$ ) did not change the significance of the relation in the treated group. No other clinical variables correlated significantly with the SMC response in this group. In addition, there was no difference in baseline blood flow or in the blood flow response in the SMC between patients with predominant left sided $(\mathrm{n}=4)$ compared with right sided $(\mathrm{n}=8)$ symptoms although it must be noted that our sample sizes were small (mean baseline blood flow in PET counts, right $=1030$ $(\mathrm{SD}=51.8)$, left $=1086(\mathrm{SD}=66.6)$; mean change in blood flow with levodopa in PET counts, right $=-3.3 \% \quad(\mathrm{SD}=4.5)$, left $=-3.1 \%(S D=8.6) ; t$ tests, p values $>0.14)$. Finally, average UPDRS baseline ratings from the left side compared with right side of the body correlated equally well with the blood flow response in the SMC within the treated PD group (right: $r=-0.69, \mathrm{p}=0.01$; left: $r=-0.68, \mathrm{p}=0.02$ ).

\section{VLPFC region}

Figure $2 \mathrm{~B}$ shows the differences in the VLPFC blood flow response to levodopa across the three groups. At baseline, the treated PD group had higher regional blood flow than the levodopa naive PD group $(t=-2.1, \mathrm{p}=0.049)$ and the controls $(t=-3.0, \mathrm{p}=0.007)$. On levodopa mean blood flow in this region did not differ between groups. However, blood flow in the treated PD group declined in this region whereas blood flow in the control group increased, and this difference was significant $(t=5.2, \mathrm{p}<0.001)$. Interestingly, the degree of change for the levodopa naive PD group was intermediate between the treated PD group and the controls, and was significantly different from both (levodopa naive PD $v$ treated $\mathrm{PD}, t=3.1, \mathrm{p}=0.005$; levodopa naive PD $v$ controls, $t=2.4$, $\mathrm{p}=0.025$ ). Within group analyses (one sample $t$ tests) revealed that the control group had a significant increase in blood flow $(t=4.4, \mathrm{p}<0.001)$ and the treated PD group had a significant decrease in blood flow in the left VLPFC region $(t=-3.0$, $p=0.01$ ). The levodopa naive PD group did not have a significant response in the left VLPFC $(t=1.1, \mathrm{p}=0.29)$ (see figs 3B and $4 \mathrm{~A})$.

Age did not correlate significantly with response in this region, either within or across groups. Furthermore, we removed four of the youngest normal controls to provide a
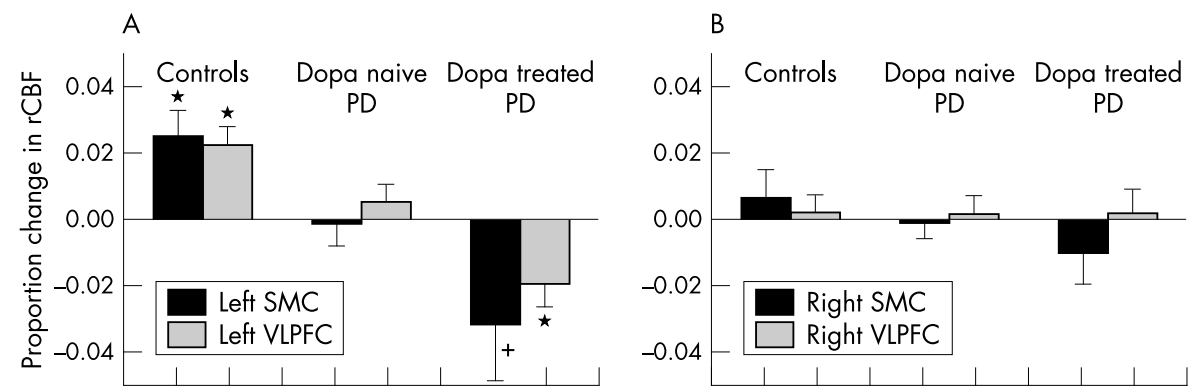

Figure 4 Change (SEM) in blood flow after oral levodopa in the (A) left SMC and VLPFC and (B) right SMC and VLPFC in controls, levodopa naive $\mathrm{PD}$, and treated $\mathrm{PD}$. Blood flow changed significantly $\left({ }^{*} p<0.05\right)$ in the control and treated PD groups in the left VLPFC and left SMC (+ treated $P D$ group non-significant change, $p=0.08)$. Blood flow did not change significantly for any group in the right SMC or right VLPFC. 
Table 2 Regional effects of levodopa on blood flow; SPM99 cluster analysis results

\begin{tabular}{|c|c|c|c|c|c|}
\hline \multirow[b]{2}{*}{ Location of cluster } & \multirow{2}{*}{$\begin{array}{l}\text { p value } \\
\text { (corrected) }\end{array}$} & \multicolumn{3}{|c|}{$\begin{array}{l}\text { Cluster level } \\
\text { Peak voxel coordinates }\end{array}$} & \multirow[b]{2}{*}{ Direction of effect } \\
\hline & & $\mathrm{x}$ & y & z & \\
\hline \multicolumn{6}{|c|}{ Differential effects of levodopa; treated PD $v$ controls } \\
\hline Left VLPFC & 0.049 & -37 & 35 & 3 & $\begin{array}{l}\text { Treated PD-decreased blood flow; } \\
\text { Controls-increased blood flow }\end{array}$ \\
\hline Left SMC & 0.036 & -39 & -23 & 47 & $\begin{array}{l}\text { Treated PD-decreased blood flow; } \\
\text { Controls-increased blood flow }\end{array}$ \\
\hline \multicolumn{6}{|c|}{ Levodopa $v$ baseline; all subjects } \\
\hline Midbrain & $<0.001$ & -9 & -23 & -25 & Increase in blood flow \\
\hline Anterior cingulate & $<0.001$ & -13 & 31 & 1 & Increase in blood flow \\
\hline Right inferior parietal & $<0.001$ & 61 & -39 & 11 & Decrease in blood flow \\
\hline
\end{tabular}

better age matched subsample for the treated PD group. This modification eliminated the age difference between the two groups, as described above, yet the difference in blood flow response to levodopa in the left VLPFC remained robust $(t=4.90, \mathrm{p}<0.001)$.

Within the treated PD group, left VLPFC response did not correlate with UPDRS scores or other clinical variables. In addition, there was no difference in baseline blood flow or in the blood flow response in the VLPFC between patients with predominant left sides $(\mathrm{n}=4)$ compared with right sided $(\mathrm{n}=8)$ symptoms although our sample sizes were small (mean (SEM) baseline blood flow in PET counts, right=1148 (15.2) left $=1163$ (18.2); mean (SEM) change in blood flow with levodopa in PET counts, right $=-1.8 \%$ (0.6) left $=-2.2 \%$ (1.7); $t$ tests, $\mathrm{p}$ values $>0.56$ )

\section{Homologous right sided SMC and VLPFC blood flow}

To determine if group differences were also present in right SMC and right VLPFC, but simply fell below our statistical threshold, we applied the left SMC and VLPFC regions to the right side of the brain (by flipping the regions defined in the SPM analysis from left to right) and obtained mean blood flow for each subject at baseline and on levodopa. We then calculated average change in blood flow induced by levodopa in these regions for each group. There were no significant changes in blood flow for any of the three groups (one tailed $t$ tests, p values $>0.27$ ) (fig 4B). However, it should be noted
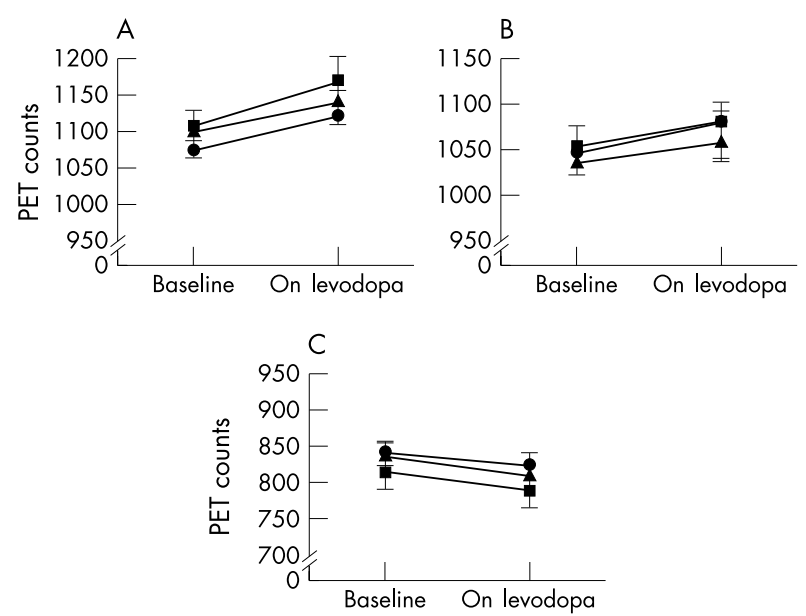

Figure 5 Means (SEM) for baseline and on levodopa blood flow in (A) midbrain, (B) anterior cingulate, and (C) right parietal for control group (circles), levodopa naive PD group (triangles), and treated PD group (squares). These three regions showed significant change in blood flow with levodopa over all subjects and within each group; there were no differences between groups at baseline, on levodopa or in the change in blood flow after levodopa administration. that because of our restricted field of view, we cannot sample the most superior slices containing SMA.

\section{Levodopa responses across all subjects}

Across all subjects, regional flow significantly increased in midbrain and anterior cingulate cortex yet significantly decreased in the right lateral inferior parietal cortex (table 2). The midbrain region was very large, extending from the upper pons to just below the thalami, covering the entire midbrain and bilateral subthalamic nuclei (fig 2C). The anterior cingulate and right lateral inferior parietal regions were comparatively restricted (fig 2C and 2D). There were no significant differences between groups in baseline, on levodopa blood flow or change in blood flow. All groups demonstrated a significant change in blood flow (one sample $t$ tests, $\mathrm{p}$ values $<0.05$ ) (fig 5).

\section{Regional blood flow in 953b compared with 961 scans} Most of our scans were performed on the 953 scanner (35 of 44). Only five normal controls, two dopa naive PD, and two dopa treated PD were scanned on the 961 scanner. Because of the small sample sizes, it is impossible to perform meaningful statistical comparisons of blood flow responses between scanners. However, we did perform a repeated measures GLM on baseline and on levodopa blood flow in the SPM identified regions with only subjects who were scanned on the 953b scanner. These analyses demonstrated that (1) the significant group $\times$ condition interaction in the SMC and VLPFC regions found in the entire sample was still present in this smaller sample (SMC: $F(1,19)=13.2, \mathrm{p}=0.002$; VLPFC: $F(1,19)=17.0$, $\mathrm{p}=0.001)$ and (2) the significant main effect of drug on blood flow in the midbrain, anterior cingulate and right parietal regions found in the entire sample was also present in this smaller sample (midbrain: $F(1,32)=71.2, \mathrm{p}<0.001$; anterior cingulate: $\quad F(1,32)=42.2, \quad \mathrm{p}<0.001)$; right parietal: $F(1,32)=33.2, \mathrm{p}<0.001)$.

\section{Levodopa and carbidopa plasma concentrations}

We were able to measure levodopa and carbidopa plasma concentrations in 38 of the 44 subjects. Levodopa concentrations peaked between 30 and 60 minutes after the oral dose of levodopa, and then remained above values found to provide symptomatic benefit in other studies for another 45 minutes (overall mean levodopa concentrations $=1302 \mathrm{ng} / \mathrm{ml}, \mathrm{SD}=598$ $\mathrm{ng} / \mathrm{ml}$ ). During this time period, collection of the on levodopa blood flow measurements occurred. Carbidopa concentrations remained stable across the study (about 500-600 $\mathrm{ng} / \mathrm{ml}$ ). There were no significant differences among groups in levodopa concentrations at the time of the on levodopa scans and no significant correlations between levodopa concentrations and the regional changes in blood flow discussed above. 


\section{Global blood flow}

All regional data were analysed using normalised PET counts, which are linearly related within a given scan to quantitative regional cerebral blood flow. ${ }^{40}$ Absolute global blood flow was quantified in 21 subjects (five controls, nine levodopa naive PD patients, seven treated PD patients). Average baseline global blood flow was $59.0 \mathrm{ml} /(100 \mathrm{~g} / \mathrm{min})(\mathrm{SD}=14.6)$ and the average on levodopa absolute blood flow was $57.9 \mathrm{ml} /(100 \mathrm{~g} / \mathrm{min})$ $(\mathrm{SD}=12.25)$. In a repeated measures general linear models analysis with group (controls, levodopa naive PD, treated PD) and condition (baseline, on levodopa) as factors, the main effects of group or condition were not significant, (group $F(2,18)=2.7, \mathrm{p}=0.10$; condition $F(1,18)=0.33, \mathrm{p}=0.58)$ and neither was the interaction between the two $(F(2,18)=0.001$, $\mathrm{p}=0.99$ ). Our finding that levodopa did not change global blood flow is consistent with other studies using adequate carbidopa pretreatment. ${ }^{14}$

\section{DISCUSSION}

This study showed that chronically treated PD patients have abnormal blood flow responses to an acute dose of levodopa in left sensorimotor cortex (SMC) and left ventrolateral prefrontal cortex (VLPFC) compared with controls. Chronically treated PD patients also responded differently from levodopa naive PD patients in these regions, but this difference was significant only in the VLPFC region. Within the treated PD group only, the SMC response was strongly associated with increased motor severity. In contrast, the VLPFC response in this group was not associated with motor severity.

The additional finding that motor symptoms correlate with the SMC response in treated but not levodopa naive PD suggests that both disease severity and chronic exposure to levodopa may be related to this abnormal blood flow response to levodopa. The availability of effective symptomatic treatment makes it difficult to separate disease severity from treatment exposure rigorously in PD patients as the more severely affected patients typically have been treated for a longer time. Interestingly, other data suggest that both long term treatment and increasing severity may contribute to the development of dyskinesias that represent an abnormal behavioural response to levodopa. ${ }^{71}$ We speculate that a similar pathogenic mechanism requiring severity and long term exposure to levodopa could interact to produce the functional abnormalities in specific dopaminergic pathways that we found in the absence of behavioural alterations. However, our study does not directly investigate this issue as none of the chronically treated PD patients in this study had levodopa induced dyskinesias.

Disease progression or chronic levodopa exposure could change the dopaminergic mediated input to SMC changing the SMC responses found in this study. The basal ganglia provide primary input to SMC and are a major site of action of dopamine. The net effect of dopamine activity in the striatum would be to reduce inhibition of internal pallidum on thalamic nuclei thereby increasing the activity of excitatory projections from thalamus to SMC. ${ }^{42}$ We postulate that the increased blood flow in SMC in controls in response to levodopa reflects the increased activity of these thalamocortical projections. The direct dopamine agonist apomorphine produced similar blood flow changes in SMC in another group of normal subjects. ${ }^{43}$ However, in chronically treated PD patients, baseline blood flow was non-significantly higher than controls, and with levodopa, blood flow decreased. Our data suggest that levodopa can decrease SMC input or local SMC activity in treated PD patients. Of course, levodopa induced changes in the pattern of neuronal firing may be more important than whether mean firing rates increase or decrease. ${ }^{44}$

The dopaminergic system also has direct and indirect connections to the prefrontal cortex that could mediate the effects on VLPFC. The ventral tegmental area and the substantia nigra, pars compacta (SNpc) have direct dopaminergic connections to prefrontal cortex. Contact between these dopaminergic terminals and dendrites of pyramidal neurons in the prefrontal cortex may allow them to modulate excitatory input. ${ }^{45}{ }^{46}$ Furthermore, both basal ganglia output nuclei, substantia nigra pars reticulata ( $\mathrm{SNpr}$ ) and GPi, project via specific thalamic nuclei to prefrontal cortex. ${ }^{42}$ GPi also sends direct inputs to lateral prefrontal cortex. ${ }^{47}$ Increased input from these pathways to the VLPFC or increased local VLPFC activity in response to levodopa may mediate the increased blood flow seen in controls. Long term treatment with levodopa could affect the response sensitivity of any of these pathways to levodopa causing a decrease in blood flow responses in the VLPFC after a dopaminergic challenge. Interestingly, the VLPFC has been implicated in a specific cognitive process, namely working memory, which is known to be deficient in PD and can be changed by dopaminergic agents. ${ }^{48-50}$

This study also reports that levodopa activates midbrain and anterior cingulate regions to a similar degree in PD and controls. As these regions responded similarly across groups, these responses probably do not reflect PD pathophysiology. Previous animal and human studies support this finding and have demonstrated that dopamine agonists increase blood flow or metabolism in these regions in normal and dopamine deficient states. ${ }^{43} 5152$ Although midbrain responses to levodopa or dopamine agonists are common in animal studies, to our knowledge our study is the first to report significantly increased blood flow in the midbrain in response to a dopamimetic challenge in humans with or without PD. Interestingly, the diffuse midbrain response seen here is similar to results obtained using 2-deoxyglucose ex vivo autoradiograms, which have a much higher resolution than our PET measures. ${ }^{51}$ We speculate that the midbrain response reflects a normal functional pathway including striatal-nigral input, pallidal input to superior colliculus and midbrain extraparamidal area and subthalamic nucleus inputs to SNpr. Levodopa's effect on blood flow in the anterior cingulate could reflect increased input from the ventral striatum or cortico-cortical connections. ${ }^{53}$ Finally, blood flow decreased in the right lateral inferior parietal region in response to levodopa across all subjects. Again, as this effect was similar across groups, it may not be relevant to PD pathophysiology. This general region has been affected by dopamine challenge in other studies. A dopamine agonist decreased baseline blood flow in parietal cortex in baboons. ${ }^{54}$ In addition, levodopa modulated parietal response to a working memory task in Parkinson disease. ${ }^{55}$ Blood flow response to levodopa in the parietal region could reflect altered activity in cortico-cortical connections, perhaps from prefrontal cortex.

The laterality of some of our findings (left SMC, left VLPFC) does not seem to be related to laterality of PD symptoms in a direct manner. However, we have seen a preferential left sided bias on effects of levodopa in the brain of other species and normal humans. Both awake and sedated normal animals (macaque and baboon) show asymmetric blood flow responses to levodopa in the putamen (left greater than right). ${ }^{14}$ In addition, we reported greater left than right putamen responses in normal humans after levodopa. ${ }^{15}$ To investigate the possibility that homologous right sided regions may have responded to levodopa but did not reach statistical significance, we examined the right SMC and right VLPFC regions in our blood flow data. We found no significant changes in either region on the right for any of the three subject groups (fig 4B). It should be noted, however, that we cannot sample the most superior slices of the brain containing SMC and so cannot draw conclusions about the entire superior-inferior extent of the SMC on either side of the brain. These results do indicate that frontal cortical regions can respond asymmetrically to a systemic levodopa challenge in both normal and PD groups. Thus, it seems highly unlikely that our lateralised results are a function of disease or disease 
severity. Although long term levodopa treatment can dramatically affect asymmetric motor responses to an acute dose of levodopa, ${ }^{56}$ no study has clearly examined neurophysiological asymmetries as a function of levodopa treatment history in PD patients.

In conclusion, we found that a dopaminergic challenge revealed normal and abnormal neural circuits in PD patients undergoing chronic levodopa treatment. We identified cortical regions (left SMC and VLPFC) that respond abnormally to levodopa in PD patients with chronic exposure to levodopa. The SMC response correlated strongly with severity of motor symptoms, suggesting that both exposure and severity modulate functional responses in this region. In contrast, the VLPFC response did not correlate with motor severity. Thus, chronic exposure to levodopa and disease severity affect the function of specific dopaminergic pathways before dopa induced dyskinesias or psychosis have developed

\section{ACKNOWLEDGEMENTS}

We thank Tom O Videen, PhD, Lee Tempel, MD, and Ann-Mary MacLeod for expert assistance.

\section{Authors' affiliations}

T Hershey, K J Black, Department of Psychiatry, Washington University School of Medicine, USA

K J Black, A Z Snyder, J S Perlmutter, Department of Radiology, Washington University School of Medicine

T Hershey, K J Black, J L Carl, L McGee-Minnich, A Z Snyder, J S Perlmutter, Department of Neurology, Washington University School of Medicine

J S Perlmutter, Department of Anatomy and Neurobiology, Washington University School of Medicine

Funding: this work was supported by NIH grants NS41248, NS32318, NS10787, NS01898, NS41509, NS39913 the Greater St Louis Chapter of the American Parkinson's Disease Association (APDA), the APDA Advanced Center at Washington University in St Louis, Alzheimer's Disease and Related Disorders Program, National Alliance for Research in Schizophrenia and Depression, a donation from Ruth Kopolow and the McDonnell Center for the Study of Higher Brain Function.

Competing interests: none declared.

\section{REFERENCES}

1 Cotzias GC, van Woert HH, Schiffer LM. Aromatic amino acids and modifications of parkinsonism. N Engl J Med 1967:276:374-9.

2 Factor SA, Molho ES, Podskalny GD, et al. Parkinson's disease: drug induced psychiatric states. Adv Neurol 1995;65:115-38.

3 Colosimo C, DeMichelle M. Motor fluctuations in Parkinson's disease pathophysiology and treatment. Eur Neurol 1999:6:1-21.

4 Engber TM, Susel Z, Kuo S, et al. Chronic levodopa treatment alters basal and dopamine agonist-stimulated cerebral glucose utilization. J Neurosci 1990;10:3889-95.

5 Kang UJ, Wu Q, Papasian N, et al. The contributions of disease severity and chronic therapy to motor response complications in parkinsonian rats. Soc Neurosci Abstr 2001;27:574.14.

6 Mones RJ, Elizan TS, Siegal GJ. Analysis of L-dopa induced dyskinesia in 51 patients with parkinsonism. J.Neurol Neurosurg Psychiatry 1971;34:668-73.

7 Ballard PA, Tetrud JW, Langston JW. Permanent human parkinsonism due to 1-methyl-4-phenyl-1,2,3,6, tetrahydropyridine (MPTP): seven cases. Neurology 1985;35:949-56.

8 Gerfen CR. Molecular effects of dopamine on striatal-projection pathways. Trends Neurosci 2000;23:S64-70

9 Schwartz WJ, Smith CB, Davidsen L, et al. Metabolic mapping of functional activity in the hypothalamo-neurohypophysial system of the rat. Science 1979;205:723-5

10 Gold L, Lauritzen M. Neuronal deactivation explains decreased cerebellar blood flow in response to focal cerebral ischemia or suppressed neocortical function. Proc Natl Acad Sci USA 2002:99:7699-704

11 Lauritzen M. Relationship of spikes, synaptic activity, and local change of cerebral blood flow. J Cereb Blood Flow Metab 2001;21:1367-83.

$12 \mathrm{McCulloch} \mathrm{J}$. Role of dopamine in interactions among cerebral function metabolism, and blood flow. In: MacKenzie ET, Seylaz J, Bés A, eds. Neurotransmitters and the derebral circulation. New York: Raven, 1984:137-55

13 Azuma H, Miyazawa T, Mizokawa T, et al. [Stimulatory effects of lisuride on local cerebral blood flow and local cerebral glucose utilization in rats]. Nippon Yakurigaku Zasshi 1988:91:341-9.

14 Hershey T, Black KJ, Carl JL, et al. Dopa-induced blood flow responses in non-human primates. Exp Neurol 2000;166:342-9.
15 Hershey T, Black KJ, Stambuk MK, et al. Altered thalamic response to levodopa in Parkinson's patients with dopa-induced dyskinesias. Proc Natl Acad Sci USA 1998;95:12016-21.

16 Melamed E, Lavy S, Cooper G, et al. Regional cerebral blood flow in Parkinsonism. J Neurol Sci 1978:38:391-7.

17 Kobari M, Fukuuchi Y, Shinohara T, et al. Levodopa-induced loca cerebral blood flow changes in Parkinson's disease and related disorders. J Neurol Sci 1995;128:212-18.

18 Perlmutter JS, Raichle ME. Regional blood flow in hemiparkinsonism. Neurology 1985;35:1127-34.

19 Leenders KL, Wolfson LI, Gibbs JM, et al. The effects of I-dopa on regional cerebral blood flow and oxygen metabolism in patients with Parkinson's disease. Brain 1985;108:171-91.

20 Montastruc JL, Celsis P, Agniel A, et al. Levodopa-induced regional cerebral blood flow changes in normal volunteers and patients with Parkinson's disease. Mov Disord 1987;2:279-89.

21 Oishi M, Mochizuki Y, Hara M, et al. Effects of intravenous L-dopa on P300 and regional cerebral blood flow in parkinsonism. International Journal of Neuroscience 1996;85:147-54.

22 Henriksen L, Boas J. Regional cerebral blood flow in hemiparkinsonian patients. Emission computerized tomography of inhaled ${ }^{133}$ Xenon before and after levodopa. Acta Neurol Scand 1985;71:257-66.

23 Black KJ, Hershey T, Hartlein JM, et al. Parkinson's disease patients with levodopa-related mood fluctuations have increased posterior cingulate response to acute levodopa. Soc Neurosci Abstr 2001 :27:785.7.

24 Folstein MF, Folstein SE, McHugh PR. "Mini-mental state": a practical method for grading the cognitive state of patients for the clinician. $J$ Psychiatr Res 1975;12:189-98.

25 Hamilton M. A rating scale for depression. J Neurol Neurosurg Psychiatry 1960;23:56-62.

26 Lang AE, Fahn S. Assessment of Parkinson's disease. In: Munsat TL, ed. Quantification of neurologic deficit. Boston: Butterworths, 1989:285-309.

27 Spinks TJ, Jones T, Bailey DL, et al. Physical performance of a positron tomograph for brain imaging with retractable septa. Phys Med Biol 1993;37: 1637-55.

28 Videen TO, Perlmutter JS, Herscovitch $P$, et al. Brain blood volume, flow and oxygen utilization measured with $0-15$ radiotracers and positron emission tomography: revised metabolic computations. J Cereb Blood Flow Metab 1987;7:513-16.

29 Herscovitch P, Markham J, Raichle M. Brain blood flow measured with intravenous $\mathrm{H}_{2}^{15} \mathrm{O}$. I. Theory and error analysis. J Nucl Med 1983;24:782-9.

30 Baruzzi A, Contin M, Albani F, et al. Simple and rapid micromethod for the determination of levodopa and 3-O-methyldopa in human plasma by high-performance liquid chromotography with coulometric detection. J Chromatogr B Biomed Appl 1986;375: 165-9.

31 Carl JL, Perlmutter JS. A new method to measure L-DOPA and metabolites in plasma. Soc Neurosci Abstr 1998;24:352.

32 Snyder AZ. Difference image vs ratio image error function forms in PET-PET realignment. In: Myers R, Cunningham V, Bailey D, et al, eds. Quantification of brain function using PET. San Diego, CA: Academic Press, 1996.

33 Ojemann JG, Buckner RL, Akbudak E, et al. Functional MRI studies of word-stem completion: reliability across laboratories and comparison to blood flow imaging with PET. Hum Brain Mapp 1998;6:203-15.

34 Talairach J, Tournoux P. Co-planar stereotaxic atlas of the human brain. New York: Theime Verlag, 1988.

35 Lancaster JL, Glass TG, Lankipalli BR, et al. A modality-independent approach to spatial normalization of tomographic images of the human brain. Hum Brain Mapp 1995;3:209-23.

36 Andersson JL, Sundin A, Valind S. A method for coregistration of PET and MR brain images. J Nucl Med 1995;36:1307-15.

37 Black KJ, Snyder AZ, Koller JM, et al. Template images for nonhuman primate neuroimaging. 1. Baboon. Neuroimage 2001;14:736-43.

38 Holmes AP, Friston KJ. Generalizability, random effects and population inference. Neuroimage 1998;7:S754

39 Friston KJ, Holmes AP, Worsley KJ. How many subjects constitute a study? Neuroimage 1999;10:1-5.

40 Fox PT, Mintun MA, Raichle ME, et al. A noninvasive approach to quantitative functional brain mapping with $\mathrm{H} 2(15) \mathrm{O}$ and positron emission tomography. J Cereb Blood Flow Metab 1984;4:329-33.

41 Boyce S, Rupniak NM, Steventon M, et al. Nigrostriatal damage is required for induction of dyskinesias by L-dopa in squirrel monkeys. Clin Neuropharmacol 1990:5:448-58

42 Mink JW. The basal ganglia: Focused selection and inhibition of competing motor programs. Prog Neurobiol 1996;50:381-425.

43 Kapur S, Meyer J, Wilson AA et al. Activation of specific cortical regions by apomorphine: an ${ }^{15} \mathrm{OH}_{2} \mathrm{O}$ PET study in humans. Neurosci Lett 1994; 176:21-4.

44 Boraud T, Bezard E, Bioulac B, et al. Dopamine agonist-induced dyskinesias are correlated to both firing pattern and frequency alterations of pallidal neurones in the MPTP-treated monkey. Brain 2001; 124:546-57.

45 Krimer LS, Jakab RL, Goldman-Rakic P. Quantitative three-dimensional analysis of the catecholaminergic innervation of identified neurons in the macaque prefrontal cortex. J Neurosci 1997;17:7450-61.

46 Goldman-Rakic PS. Dopamine-mediated mechanisms of the prefrontal cortex. The Neurosciences 1992;4:149-59. 
47 Middleton FA, Strick PL. Anatomical evidence for cerebellar and basal ganglia involvement in higher cognitive function. Science 1994;266:458-61

48 Sawaguchi T, Goldman-Rakic PS. The role of D1-dopamine receptor in working memory: local injections of dopamine antagonists into the prefrontal cortex of rhesus monkeys performing an oculomotor delayed-response task. J Neurophysiol 1994;71:515-28.

49 Gabrieli JDE, Singh J, Stebbins GT, et al. Reduced working memory span in Parkinson's disease: evidence for the role of a frontostriatal system in working and strategic memory. Neuropsychology 1996;10:322-32.

50 Luciana $M$, Depue RA, Arbisi P, et al. Facilitation of working memory in humans by a D2 dopamine receptor agonist. J Cogn Neurosci 1992;4:58-68.

51 Trugman JM, Wooten GF. The effects of L-DOPA on regional cerebral glucose utilization in rats with unilateral lesions of the substantia nigra. Brain Res 1986;379:264-74.
52 Grasby PM, Friston KJ, Bench CJ, et al. The effect of the dopamine agonist, apomorphine, on regional cerebral blood flow in normal volunteers. Psychol Med 1993;23:605-12.

53 Alexander GE, Crutcher M, DeLong M. Basal ganglia-thalamo-cortical circuits: Parallel substrates for motor, oculomotor, "prefrontal," and "limbic" functions. Prog Brain Res 1990;85: 119-46.

54 Black KJ, Hershey T, Koller JM, et al. A possible substrate for dopamine-related changes in mood and behavior: prefrontal and limbic effects of a D3-preferring dopamine agonist. Proc Natl Acad Sci 2003;99:17113-18.

55 Mattay VS, Tessitore A, Callicott JH, et al. Dopaminergic modulation of cortical function in patients with Parkinson's Disease. Ann Neurol 2002;51:156-64

56 Carey RJ. Motoric sensitization and levodopa accumulation after chronic levodopa treatment in an animal model of Parkinson's disease. J Geriatr Psychiatry Neurol 1993;6:152-60.

\section{Applications are invited for the post of Editor}

Journal of Neurology Neurosurgery and Psychiatry

JNWP is one of the premier journals in neurology. We now seck an editor who can increase its scientific and international reputation while maintaining its relevance to practising neurologists woeldwide.

Applications from specialists in any branch of neurology, neurosurgery or psychiatry are imvited. Applications from outside the UK are encouraged and joint applications from two or more individusls willing to act as a team are welcomed.

Full editcrial support and training will be provided. The jaurnal will fund any necessary locum requirements. The successful applicant(s) will be free to select his/her own team of associate editors and board members.

Closing date for applications is 1st August 2003. Interviews will be held on 9 th September 2003 . It is envisaged that the outgoing editor will gradually hand over during the fourth quarter of 2003 and the successful candidate will officially take up the post of editor in January 2004.

Further details of the post can be discussed with the publishing director, Mrs Alex Whlliamson and/or the retiring editor Professor Christopher Kennard (Tel +44 (0)20 8946 7598; email ckennardesic.acuk). A job description is available on request from Mrs Williamson.

Applications should be sent to Mrs Alex Williamson, BMs Publishing Group, BMA House, Tavistock Square, London WC1H 9.R, UK. Tel +44 $(0) 207383$ 6069/6169; Fax +44 (0)20 7383 6668; email awilliamson Sbmjgroup.com

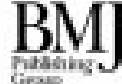

